\title{
Adaptation Studies through Screenplay Studies: Transitionality and the Adapted Screenplay Jamie Sherry
}

Analogous to screenplay studies' relatively recent theoretical progression from manuals and rudimentary narratological and structural theories, contemporary adaptation studies has also transcended its origins in the research and pedagogy of English literature. However, unlike screenplay studies, recent adaptation theory has attempted to both conceive of, and critique, the notion of itself as a field of study, in order to better understand what this entails, and how this may affect its future relevance. Most notably, contemporary adaptation studies has vociferously rejected the fidelity-led approach to adaptation, in which a media text (film, television, theatre, etc.) derived from (usually) literature is examined and understood through a comparative paradigm that privileges the authority of that literary source. A multitude of recent adaptation studies texts both build upon, and refute, theories presented in George Bluestone's seminal Novels into Film (1957), to fully discredit a methodology that is widely deemed to be both unscholarly and elitist in its logocentric prioritising of the printed word. In fact, it is difficult to find any recent academic texts on adaptation that avoid systematically propagandising the many shortcomings of a critical approach that confers immediate status on an originating text, or that uses 'closeness' or 'faithfulness' as a criterion for success. The theories of notable adaptation theorists, including Deborah Cartmell and Imelda Whelehan (1999, 2010), Kamilla Elliott (2003), Robert Stam (2004), Thomas Leitch (2005, 2007), Linda Hutcheon (2006), Christine Geraghty (2008), and Simone Murray (2011), amongst many others, have all but rejected the dominance of fidelity-based discourses rooted in compare-contrast case-studies. In journals, and an ever expanding set of theoretical monographs and edited collections, the field of adaptation studies has attempted to move beyond these models of traditional comparative analyses. Whilst this comparecontrast approach may tell us a great deal about one discrete adaptation project, it often fails to interrogate or illuminate broader and more general concepts of adapting in contemporary media culture. More helpfully, post-fidelity adaptation studies has seen the incorporation of a number of interesting and expansive research subjects, including poststructuralist and postmodern concepts of authorship, intertextuality and transtextuality, appropriations and borrowings, fan cultures and audience studies, reception theory, convergence culture and cross-platform transmedia and digital storytelling modes, amongst others. 
Despite these welcome developments in adaptation studies, this article will question why the field has almost completely ignored the importance of various production modes and texts that remediation produce, and will focus on the adapted screenplay as one area of neglect. Conversely, it will also highlight the tendency for screenplay studies to overlook the fact that the vast majority of screenplay projects are adaptations, or remediations of some kind, and how this affects both the theorising of the screenplay, and the practice of the adapting screenwriter. Literary adaptations, in particular, not only form a very high percentage of produced and distributed films per year (and an especially high proportion of prestige productions and Academy Award winners), but it has also been argued that all films are 'adaptations' due to the fact they remediate previous works intertextually, and the screenplay specifically (Stam 2004: 45). This study is also a response to cinema's increasing tendency to seek refuge in precursory texts, whether they are in the form of adaptations, re-adaptations, re-boots, sequels, prequels, franchises or remakes. As Steven Price notes, the prevalence of adapted films is unquestionable, constituting 'more than four-fifths of Academy Awards for Best Picture, and fourteen out of the twenty highest-grossing pictures of the twentieth century' (Price 2010: 54). This article will also examine the extent to which contemporary adaptation and screenplay studies share many theoretical approaches to the process, production and reception of various media, whilst also considering the inherent transitionality of contemporary screenwriting. It will also posit some fruitful areas of mutuality and cross-over that could be potentially beneficial to those scholars working in either or both fields.

\section{SCREENWRITING STUDIES AND ADAPTATION STUDIES}

Contemporary screenwriting studies corresponds closely to the field of adaptation studies in a number of notable ways. Both fields have expanded and formalised themselves relatively recently, through a conflation of research, pedagogy and practice. They also share a notably transdisciplinary grouping of academics from various studies of literature, film, theatre and production, keen to illuminate their own research preoccupations through a variety of approaches and theories. Each field of study has formalized and loosely homogenized themselves via of a set of formal conferences and symposiums, journals and book series, whilst also drawing on a small number of older, foundational works that become recontextualized within new paradigms and theoretical frameworks. Whilst screenplay studies has much it can learn from adaptation studies, it is notable that the latter has largely ignored the industrial and financial contexts of film and television production. Adaptation studies traditionally 
favours analysis of fixed, canonical works over creative process, and is biased towards unified media such as the source novel, and the final adapted film, over the adapted screenplay that exists between them. Indeed, it is notable that various scholars of adaptation concern themselves with natural questions of authorship in adapted cinema, yet routinely fail to integrate the screenwriter or debates of authorship found in screenwriting theory. Furthermore, while adaptation theorists such as Stam question the devalued cultural capital of cinema in comparison to literature, and use this as a partial explanation for fidelity discourse, there is little consideration of the even 'lowlier' form of the screenplay.

Despite these shortcomings, there are some rare but useful examples of adaptation theory's application to the issue of adapted screenwriting, most notably Simone Murray's especially thorough book The Adaptation Industry: The Cultural Economy of Contemporary Literary Adaptation (2013). Murray's work is unusual in its focus on the adaptation process as a negotiation of book publishing rights, production companies, industry practitioners, post-production marketing and the many complex financial and copyright issues that are involved in adaptation projects. Usefully, Murray spends some time on the subject of screenwriting and the screenplay, noting that the media industry's relegation of the screenwriter is echoed in 'academic literary and film studies' long-standing, studied obliviousness to the screenwriter' (Murray 2011: 132). Whilst examining the financial and rights contexts of the adapted screenplay, an under-studied area of screenplay theory, Murray also notes cultural capital influenced academic responses to screenwriting that have been well explored by numerous screenplay scholars:

Breaking down neatly upon medium-specific lines, literary studies found the innately intermedial and often collaboratively written screenplay impossible to square with the Romanticised figure of the solitary author that had been enshrined in the discipline's very theoretical and methodological foundations. (Murray 2013: 132).

Pre-dating Murray, Jack Boozer's edited collection Authorship in Film Adaptation (2008) offers a detailed and thorough overview of adaptation and writing through theories of authorship, although the chapters within tend to rely on the comparative case-study analysis of texts that adaptation studies is best known for. Despite these two formative texts there is a paucity of critical material dealing with adaptation process and creative and industrial contexts for remediation.

An outcome of the banishing of fidelity-based discourses in adaptation is an alignment with certain schools of literary theory that seek to illustrate the pervasive presence of unofficial precursor texts in all works, including (but not exclusive to) film and television adaptations. In particular, the wide-spread utilisation of poststructuralist theory has highlighted issues of authorship and the need to 
undermine the dominance of the source text or author, by recognising the many paratexts and intertexts that bear influence on adaptations. Usefully for screenplay studies, this displacement of the authority of the source and author naturally encourages, or at least creates a space for, the study of the adapted screenplay. As screenplay studies has outlined so well, the screenplay has been commonly regarded as a functional device used to facilitate and guide film, and as a 'blueprint' or 'template' for practitioners to work from, but not adhere to. As Sternberg (2007), Boon (2008), Maras (2009), Price (2010), Nelmes (2011) and various scholars of contemporary screenplay studies have so carefully argued, the screenplay has traditionally been viewed as a functional device, reduced to an on-set guide for actors and the director. Indeed, Boon argues that the screenplay 'has received far less attention in scholarly circles than in popular culture' (Boon 2008: 27), something that is especially problematic (or noticeable) when the cinematic text is an adaptation. Boon continues that whilst 'mention of the screenplay occasionally makes its way into film scholarship,' it is invariably 'overpowered by examinations of the film or a preceding work, such as a novel or a play that the screenplay has adapted for film' (Boon 2008: 27). Due to the fact that this tendency to undermine or reduce the importance of the screenplay is found in the fields of both film and literary studies, consequently the role of the adapted screenplay has become demoted or otherwise ignored in adaptation studies. Whilst it is clear that the teleplay, shooting script and the drafts of screenplays produced during adaptation function as important bridging tools and intermediary texts, they are also discrete literary works, with their own poetic qualities.

That adaptation scholars rarely study the screenplay, except when it is framed as a peripheral paratext to illuminate some other part of the filmmaking process, is evidence of a critical failure to appreciate the distinct features of the screenwriting that screenplay theorists are currently engaged with. Conversely, for screenplay theorists to consider the role of screenwriting and the screenplay and not focus on the creative and industrial contexts for adaptation on some level is evidence of a failure to understand the dominance of remediated films within the industry and marketplace. Whilst there may be a preoccupation with 'the relationship between the literary source text and resultant film text' there is ultimately a need 'to adequately interrogate the complex industrial and creative processes that take place during adaptation' (Sherry 2014: 87). The lack of attention to the crucial processes of adaptation, and the transitional mode of adaptation screenwriting both creative and industrial, illustrates a fundamental gap in adaptation studies and screenplay studies, as well as offering mutually beneficial avenues for future research and pedagogy. 
The scarcity of critical analyses of the screenplay and screenwriting by adaptation theorists is all the more remarkable given both its fundamental role in the adaptation process, and the inclusion of the screenplay in some early adaptation theory. In particular, Bluestone utilised the adapted screenplay in order to 'correlate the film with the novel' using a system that recorded changes by 'imposing the shooting-script on the book' (Bluestone 1957: ix) and recording the differences:

By evolving an exact record of alterations, deletions and additions of characters, events, dialogue, I was able to reduce subjective impressions to a minimum. The method calls for viewing the film with a shooting-script at hand. During the viewing, notations of any final changes in the editing were entered on the script. After the script had become an accurate account of the movie's final print, it was then superimposed on the novel. (Bluestone 1957: ix)

For Bluestone, this method allowed him to enter into a critical case-study evaluation with an 'accurate and reasonably objective record of how the film differed from the book' (Bluestone 1957: ix). However, naturally this method is fraught with a number of theoretical and textual problems that greatly compromise the objective analysis of an adapted film to its source. Firstly, as screenplay scholars have collectively demonstrated, the screenplay is an unstable document, with multiple versions and, whilst the shooting script is deemed to be the final and definitive document used on set, it is also liable to changes throughout the shoot. Secondly, this type of analysis assumes that the production of a film (including post-production editing) slavishly adheres to the shooting script. Furthermore, in this early work Bluestone also fails to account for elements specific to the screenplay, particularly the expressionistic and/or economical and manifest-orientated language of the film script. Whilst this attention to the adapted screenplay is noteworthy, it does highlight its status in adaptation studies as a 'blueprint' for a film, rather than as a shifting, transitional, literary form in its own right.

Adaptation studies has much to gain from considering the study of the intermedial screenwriting process, and the adapted screenplay should offer rich material for those scholars preoccupied with issues of media equivalence versus media specificity that aid or problematize remediation. However, its relative dismissal can be explained not just by the assumed literary value of the screenplay, but exacerbated by its association with literary source material. The adapted screenplay 'can thereby seem doubly inferior' due to its position as 'both . . . derivative and (usually) translated into a form that carries less literary value than the source story' (Price 2010: 54). Whilst literature can be studied for its use of cinematic language to convey visual, filmic stories, likewise cinema can be seen to utilise an impressive arsenal of literary, narratological devices. These demarcations and subjective analyses enhance our understanding of the types of ideologies that choose to remove the screenplay text from 
serious analysis. The screenplay, like the theatre play, does not generally contain the kind of firstperson character psychologies and complexity of language and emotion at which literature tends to excel. Also, it does not offer the aesthetic pleasure gained from the visceral experience of the audiovisual spectacle of cinema due to its very nature as a literary form that conveys and implies the filmic form. The textual analysis of the screenplay sees it framed within a discourse of its function as it becomes compared to its 'intended' media, rather than being recognised as a discrete form with its own literary and poetic properties. For instance, whilst there are isolated examples, very few scholars of film, literature, screenwriting or adaptation consider unproduced screenplays, unless stalled projects or what Murray would refer to as 'phantom adaptations' (Murray, 2008 ). This is in significant contrast to the traditional approach to the theatre play in literary studies in which its pedagogical purpose transcends its destination media, becoming studied as a distinct work with specific textual properties.

Whilst screenplays are constructed using scene headings (place and time), as well as occasional allusions to various camera angles and cuts, arguably one of the most essential elements of the screenplay is the presentation of audio, in the form of dialogue, as well as diegetic and non-diegetic sound. Therefore, it would seem to be a relatively simple and equivalent process to transpose dialogue from literature to screenplay as there is a clear audio-visual equivalent that makes the translation of these elements entirely smooth. Indeed, many adaptations will jettison large amounts of plot, but will often retain notably faithful sections of dialogue, even in relatively unfaithful projects. The screenplay form can also transpose the movement of characters, place, location or other elements of physical action with comparative ease. However, the third issue, or the 'problem' and 'challenge' for adaptors, is of course the specificity of literary devices found in first-person, conscious, internal narration; or the third-person, omnipotent narrator with their expositions on the thoughts, musings and unconscious compulsions of various characters. In order to meet this challenge, and with the advent of sound cinema, voice-over quickly became a device that adapting screenwriters utilised to present these firstperson narrations. Even non-adaptations adopted voice-over to convey thoughts, memories, back-story and exposition, particularly utilised in genre films, and film noir. The voice-over is used to great effect in the reflective, post-mortem perspective of the screenwriter protagonist Joe Gillis (William Holden) in the original screenplay for Sunset Boulevard (Billy Wilder, 1950) by Charles Brackett, Billy Wilder and D. M. Marshman, Jr. This past-tense narration device is also recycled and remediated for the same narrative purposes in Alan Ball's original screenplay for American Beauty (Sam Mendes, 1999). The use of voice-over in screenplays for adapted and non-adapted cinema reflects this tendency towards the equivalent transposing of psychological processes found in literature. This counter-intuitive tendency 
of cinema to integrate these literary narratological conventions bears the traces of both the influence of literature on film, and literary devices on adapting and non-adapting screenwriters. More importantly, the questioning of the integration of literary technique in film is an inquiry that does not fully acknowledge the process of screenwriting, and the recognition of film story as stemming almost always from a literary medium. The tendency for film critics to condemn literary elements in adaptations due to its betraying of the visual, cinematic qualities of film serves to underline the lack of awareness that the form is born from literature, in the form of the screenplay. The specific, linguistic tendency to consider the source novel as alchemically turned or transformed into a film, usually through the decisions of a director, does a disservice to the creative processes of adapted screenwriting, and the transitional processes of this form of writing.

\section{HISTORICAL/INDUSTRIAL CONTEXTS FOR THE SCREENPLAY IN ADAPTATION}

The story of authorship and film should not be told without substantial recognition of the role of adaptation in the long history of film storytelling. Conversely, the story of adaptation also requires an acknowledgment of the substantial role of screenwriting, and the contribution of the screenwriter. The development of the profession of screenwriting, and the industrial role of the screenwriter, is inextricably linked with early cinema and its immediate and widespread reliance on literature as source material. Contrary to the popular opinion that literary adaptations are predominantly a post-WWII phenomena, the birth of narrative film saw cinema turn to novels, short stories and serialised narratives with both enthusiasm and an awareness of its economic benefits. Furthermore, rather than experiencing a one-way and rigid discourse with literature, cinema actually influenced the content and publication of literary works from the moment that film technology allowed the production of films with clear narrative stories. Virginia Woolf famously viewed the relationship between literature and film as parasitic and exploitative, using dramatic analogies of murder to describe how cinema 'largely subsists upon the body of its unfortunate victim' (Woolf 1926: 315). However, in contrast to Woolf's assumption that 'cinema fell upon its prey with immense rapacity' (Woolf 1926: 315), it can be argued that the relationship between the forms was mutual and reciprocal. Despite this, the critical tension that exists between literature and film emerges at the moment cinema progressed beyond an experimental photographic form at the turn of the twentieth century. Price notes that France 'had coined the term films d'art in the early 1900s, but this designated not the quality of the films themselves but merely of their source materials' (Price 2010: 2). This 'film d'art' cinema constituted classic literature adaptations 
'despite the impossibility of reproducing either the language or more than a fragment of the plot' (Price 2010: 2). Attempts by early cinema to reproduce novels, short stories, plays and non-fiction media articles, were problematised by inadequate technology that resulted in a significantly shorter runtime than the standardised form that was established by the 1920s. Furthermore, rather than being bestowed with literary prestige by association (as tends to be the case with contemporary adaptations of classic literature) early film adaptations were termed 'picturizations' - an expression that implies the rejection of the printed word in favour of the purely visual. Another source of antagonism came in the form of undeveloped licensing laws. Early cinema was able to take advantage of ' $[\mathrm{t}]$ he relatively lawless state of copyright in America at this time,' which ultimately 'exacerbated the hostile relations between cinema and literature,' until 1911 when 'a US Supreme Court ruling ended the cannibalisation of published source material that had previously had no protection' (Price 2010: 2).

Within adaptation studies' discourse on the relationship of literature to cinema in early film there is notable lack of attention to the role of the adapted screenplay. Therefore, to understand the adapted screenplay form it is necessary for adaptation theorists to evaluate the industrial position of the text as an inherently fluid work existing in many drafts and versions within the development of film. Whilst a film production may create, reject and revisit various screenplay artefacts written prior to and during filming, the status of the screenplay is such that these documents are almost always project-specific, with little in the way of an afterlife beyond editing and distribution. In other words, their functionality is related entirely to the film they are deemed to facilitate, and the moment their use is fulfilled, these documents tend to be 'moth-balled' like so many other documents and paratexts generated during preproduction, principal shooting, and post-production. The shifting presence and influence of the screenplay provokes discussion about the disposability of the form particularly when distinguishing between the remake of an adapted film, versus the re-adaptation of the source material. Notably, in the production of adaptation remakes, the original adapted screenplay is not utilised, but instead the source material is either re-adapted or a new screenplay is optioned. Often, remakes will deliberately distance themselves. A notable and unusual deviation from this exclusion of a previous screenplay can be found in Gus van Sant's postmodern 'remake' of Psycho (1998). This adaptation not only utilises the original Joseph Stefano screenplay for Psycho (1960), but also incorporates a scene from the script omitted from Hitchcock's film due to censorship laws. Whilst the decision to re-use Stefano's script can be interpreted as conforming to the postmodern, over-faithful remake agenda of van Sant's film, it also highlights the typical rejection of the screenplay in the production of remakes. In this specific case, it serves to refocus van Sant's refusal to re-adapt Robert Bloch's original Psycho novel (1959) in his 
pursuit of ironic, cinematic authenticity. To that extent Stefano's adapted screenplay becomes an important and pivotal document with which to 'remake' Hitchcock's Psycho in the truest sense of the word, unencumbered by the cultural dominance of the source novel, or the conventional tendency to leap-frog a previous adaptation in pursuit of literary authenticity.

One of the reasons that screenplays from previous projects are not utilised in re-adaptations is partly explained by the form's assumed disposability, exacerbated by what is regarded as functionality of language, and lack of literary rhetoric and poetic expressionism. These debates provoke scrutiny of the function of prose fiction contrasted against the screenplay, and upsets received notions of their literary aims. For instance, some genre fiction can offer little in the way of a protagonist's existential angst, poetic 'literary' language or subtextual metaphor, instead focussing on the linear movement of plot and drama. Conversely, non-adapted, original screenplays for films such as Bergman's Through a Glass Darkly (1961) and Jane Campion's The Piano (1993) utilise a host of techniques and tonal qualities deemed to be 'literary' in nature. Despite this, and in the case of Bergman specifically, the limitations of the screenplay became problematic, forcing him to incorporate 'novellas, scripts and copious notes to prepare himself,' as he 'recognised the impossibility of the written form as the basis for a film' (Macdonald 2004: 266). Furthermore, Larry McMurtry claims that 'to this day there has been no more cinematographic novelist than Flaubert, who worked in blissful ignorance of it' (McMurtry 1987: 80), whilst an array of modernist and postmodernist writers, including Hilda Doolittle and Brett Easton Ellis, have incorporated the visual syntax of cinema into their writing. Boon cites Carl Dreyer's highly influential screenplay for The Passion of Joan of Arc (1928) as a profound example of the screenplay moving from 'the practical necessity to a literary form in its own right' (Boon 2008: 11).

These debates concerning the division between re-presenting elements of the original adaptation, and revisiting source material can tend to dwell on the elements of the previous adaptation that have bled, whether consciously or unconsciously, into the re-adapted text. For Boon, the distinction between adaptations and intertexts is troublesome because the "point where derivation becomes adaptation is nebulous when we approach the boundaries between the two' (Boon 2008: 195). This resituating of the mode of 'adapting' forces us to question the very act of adaptation, while the role of the industry creatives involved in re-presenting a text in a different media (whether it be a play, novelisation, video game, graphic novel, or novel) must be assessed with a different set of critical apparatus. In particular, rather than being seen as 'adaptation' per se, film-to-film adaptations tend to be regarded as remakes, 
re-imaginings, or intertextual homage. The categorisation of 'adaptation' is actually heavily weighted towards remediation - in which a text is clearly transformed into another medium, rather than transferred intermedially within its own form, such as remakes, or adaptations of short films to feature films. 'This refocusing of adaptation process forces us to reassess the conscious and unconscious decision-making of screenwriters and filmmakers invoking and alluding to various precursor texts. However, this open, intertextual approach to understanding and critically evaluating adaptation could be seen as a misunderstanding of the industrial and legal means by which texts are endowed with adaptive status. For Boon, the distinction between 'real' and 'implied' adaptations is clear: 'Unlike adaptations, an original screenplay has no easily identifiable textual precedent' and therefore 'this distinction has more to do with legal issues of ownership than with creative issues' (Boon 2008: 194). Whilst adaptation theorists may interrogate the expanding definitions of remediation within an intertextual discourse, the film industry, and screenplay theorists, set noticeably clearer, industrial and legal boundaries regarding the extent to which cinema integrates, alludes to, pays homage to, parodies, alludes, or adapts, source material.

\section{ADAPTATION SCREENWRITING: TRANSITIONALITY AND AUTHORSHIP}

The contextualising of the screenplay as a non-static form that exists in multiple drafts, in a state of flux, implies a sense of animation. As I and other screenplay theorists have argued, the adapted screenplay is a dynamic form, and a medium that often resists clear authorship, either assigned or intertextual. The study of intertextuality in adaptation has its origins in literature studies, and therefore assumes inspiration and cultural association, and fetishizes high-status literary allusions, appropriations and borrowings. In doing so it often completely ignores the far more anchored textual specificity of the adapted screenplay that exerts its obvious influence on any scripted adaptation. For two fields of study that concern themselves with issues of authorial voice, ownership, and the changing modes and methods of story, this transitional, in-between stage is both understudied and potentially fruitful.

For Wolf Rilla, the screenwriter is 'less than a writer, because his work can never be exclusively his,' whilst conversely he is also 'more than a writer because his responsibility extends past and beyond the act of putting words down on paper [sic]'ii (sic) (Rilla 1973: 15). In this sense, whilst the screenwriter has no ultimate control over the treatment of their script (unless they are a writer-director), they have created a document that transcends its own completeness. The screenplay is a text that exists to be interpreted and remediated (comparable to the theatre play and sheet music), and should be 
distinguished from literary texts such as the novel and short story that can be potentially adapted. Of all the metaphors used to describe the screenplay's function to become what Pasolini described as 'another structure' and to facilitate the film text as an intermediary form, the 'blueprint' metaphor appears to be the most common. Drawing upon the notion of an architectural plan to facilitate another structure, the blueprint metaphor is widely used in both screenwriting manuals, and critical works. Sternberg recognises that the screenplay as a blueprint for a film 'is the classic metaphor used to characterize the function and the significance of the screenplay during the production process' (Sternberg 1997: 50). However, the blueprint metaphor conveys a sense of a plan, or a purely functional document that facilitates an understanding of the structure, without any of the texture. Screenwriters have been known to conform to this restricted creative ambition, and in doing so 'have come to accept - and seek solace in - the rationale that screenplays are merely blueprints, not finished works, and are therefore not worth serious authorial investment on their part' (Kohn 1999: 443). The received concept of the screenplay as a 'blueprint' illuminates various assumptions about the functionality of the screenplay that is useful to both adaptation and screenplay studies. Whilst connoting sparseness and transitionality, the blueprint metaphor can also problematically communicate a sense of architectural authority in the destination form, limiting the possibilities for structural interpretation that an adapting screenwriter would require.

Within these debates is a lack of attention to the specific properties of the adapted screenplay, and the extent to which the blueprint metaphor fails to account for the specificity of a remediated script. One of the ways that scholars of both adaptation studies and screenplay studies can understand the unique position of the adapted screenplay is to consider it as an interstitial text; a liminal entity that falls between two modes of storytelling. The concept of liminality was made prominent by British cultural anthropologist Victor Turner, who built on the theories of Arnold van Gennep to explain the three stages of an individual's 'rites of passage' (1909). These phases of liminality, of particular relevance to the adapted screenplay, consists of the pre-liminal phase (separation), the liminal phase (transition) and finally a post-liminal phase (reincorporation), to illustrate three important phases of change in human development. Famously, Turner noted that in anthropological terms humans go through a period of adolescence in which they are '[1]iminal entities' who exist 'betwixt and between the positions assigned and arrayed by law, custom, convention, and ceremonial' (Turner 1969: 95). The concept also refers to states of suspension, for instance a text that falls between received genre classifications. However, it also adheres to a concept of the transitional, highlighting those 'in-between situations and conditions" that encourage "the dislocation of established structures, the reversal of 
hierarchies and uncertainty regarding the continuity of tradition and future outcomes' (Horvath, et al. 2009: 1). In this essay Turner expounds on this theory, arguing that:

liminality represents the midpoint of transition in a status-sequence between two positions, outsiderhood refers to actions and relationships which do not flow from a recognized social status but originate outside it, while lowermost status refers to the lowest rung in a system of social stratification in which unequal rewards are accorded to functionality differentiated positions.

(Turner 1967: 23).

Whilst adapted screenplays are read as static, literary documents, they also exist between their source media and their destination form. The screenplay's various undefined components of the source, which can include plotting, narrative, dialogue, etc. (separation), before entering a liminal phase in which the screenplay is conceived and written (transition), and then finally utilised during the post-liminal film production phase (reincorporation).

So, in adapted works the screenplay becomes the liminal, bridging text, as well as a system of transference to enable the converting of (usually) one media form into another, and functioning as a work oscillating between the source media and its destination form. One of the benefits of this concept for both screenplay studies and adaptation studies is the resituating of remediation process as taking place within the creative and industrial processes of adaptation. It also forces us to question what it is exactly that screenwriters, directors, actors, editors and other practitioners are actually adapting. Leitch outlines a set of specific questions that deal with this issue, and to highlight assumptions made about the study of adaptations, related to the source object:

Why has the novel, rather than the stage play or the short story, come to serve as the paradigm for cinematic adaptations of every kind? Given the myriad differences, not only between literary and cinematic texts, but between successive cinematic adaptations of a given literary text, or for that matter between different versions of a given story in the same medium, what exactly is it that film adaptations adapt, or are supposed to adapt?

(Leitch 2005: 150).

Leitch continues with his questioning regarding the differences between adaptation, intertextuality, and transtextuality, asking 'how does the relation between an adaptation and the text it is explicitly adapting compare to its intertextual relationships with scores of other precursor texts?' (Leitch 2005: 150). These types of hypothetical questions do little to clear up some of the issues related to the narratological intricacies of adaptation process, but they can force practitioners and theorists of adaptation to consider the processes of adaptation that could include the screenplay. The move towards a more inclusive, industrial process view of adaptation should include all of the various texts produced 
in that interim stage - indeed Boozer has noted the necessity for a 'revised contemporary sensitivity to adaptive film authorship' that should 'also include the environments of all three texts - literary, script intertext, and film' (Boozer 2008: 24). Stam is also sympathetic to the attendance of a multiplicity of sources, positing that although 'adaptation studies often assumes that the source texts are literary, adaptations can also have subliterary or paraliterary sources' (Stam 2004: 45). On this occasion Stam is specifically discussing such 'subliterary' media as comic books and computer games, to differentiate them from traditional literary source material. However, Stam also elucidates on the idea that all cinematic works are adaptations observing 'that virtually all films, not only adaptations, remakes, and sequels, are mediated through intertextuality and writing,' and most interestingly argues that '[e]ven non-adaptation fiction films adapt a script' (Stam 2004: 45). Therefore, the process of adapted and non-adapted filmmaking is ultimately transitional, so how "is a film's relation to its literary source different from its relation to its screenplay?" (Leitch 2005: 150). Price later echoes both of these points, and notes the various texts involved in adaptation:

Each version of a screenplay adapts a previous one; similarly, a film is an adaptation of the screenplay, while every adaptation is also an interpretation of one or more source texts that are amenable to other interpretation $[\ldots . .$.$] . This is likely to prove a much more fruitful approach$ than regarding the film as the creation of a single auteur [...] although the question of precisely what it is that is adapted is not always easily answered.

(Price 2010: 54).

Despite his echoing of Leitch's point about the difficulties of determining the specificity of what is being adapted, Price's position raises the issue of the screenplay as credible source material to be adapted into film, as distinct from its typical role as a document that merely facilitates film. This resituating of the screenplay as an open, transitional, interpretable document undermines the specificity of the blueprint metaphor.

Contemporary theories of adaptation offer useful ways to interrogate notions of authorship, agency, and process in screenwriting. But, conversely, the utilisation of screenwriting theory to help us better understand contrasting views of adaptation and intertextuality is less obvious, and can become fraught with a number of critical assumptions about what screenwriting is, and what the screenplay can tell us. For Boozer, '[f]ocusing on the screenplay in adaptation necessarily foregrounds issues of authorship' at a time when the field is 'weighted toward semiotics, poststructuralism, and broadly conceived influences of cultural intertextuality' (Boozer 2008: 1). This move away from close textual analysis, towards more theoretical notions of intermedial discourse in adaptation studies, has the potential to undermine the critical necessity to study the screenplay, or conversely to resituate it as a 
more important text than the source material. Boozer continues, arguing that the film script can act as way to understand the process of adaptation, as the 'composition of the screenplay illuminates the evolution of ideas that will determine the film production's relationship to its source text' (Boozer 2008: 1). Price is more specific, underlining the extent to which the unstable nature of the screenplay mirrors contemporary, poststructuralist notions of adaptation. Perhaps unconsciously alluding to Christine Geraghty's evocative 'ghostly presence' (Geraghty 2008: 195) of precursor texts, he notes that '[a]daptation offers the most familiar illustration of the play of presence, absence, and ghostliness that surround film and screenplay alike' (Price 2010: 53). Price's perspective on the neglect of the screenplay in adaptation studies highlights the apparent shortcomings of adaptation theorists. In his discussion of Leitch's views on the lack of psychological exposition in Dashiell Hammet's prose, Price notes that:

.......] it is characteristic of Leitch's approach, and indeed of adaptation studies in general, that the argument is conducted in relation to films and prose fiction but without consideration of the readerly affects that a comparably paratactic style of writing generates in the screenplay text.

(Price 2010: 57).

These 'readerly effects' relate to the lack of attention by adaptation studies to the idiosyncratic, poetic form of the screenplay, marked by similar economies of language found in poetry and the restrained syntax of some prose writers.iii

For Boozer, the 'closed fixation only on literary source and finished film' is further evidence of endemic 'indifference to the evolving intentions of producers, writers, and directors and their shifting levels of input and authority' (Boozer 2008: 3). Conversely, in literary studies the widespread research of the many documents that provide further contexts on the production of literary works, including early drafts and, in particular, editorial contributions, is an established form of scholarly research. Despite this, the amount of texts that are generated in the production of literature cannot compete with the number of different documents, volumes of multiple drafts and types of scripts produced during the development of an adapted screenplay. But what are the benefits of the study of these development documents, early drafts and paratextual ephemera, and why should they be analysed at all? For Boozer, the study of the screenplay in adaptation is a scholarly necessity if we are to understand the genealogy, or genetic development, of an adaptation from conception to final film:

A critical approach to adaptation that recognizes authorial desire through the script intertext as the film can reveal - like the many sketches a sculptor might draw in preparation for completing a statue - the significant stages of smaller decisions that finally add up to the whole.

(Boozer 2008: 24). 
The 'whole' alluded to here creates its own set of theoretical and textual problems. Certainly, it is impossible to fully know and understand all of the elements that go into the production of an adapted film, or these 'smaller decisions' that ultimately ripple through the decision-making process. However, a more pertinent question may be one that asks why scholars or critics should strive to incorporate all of these elements that constitute the 'whole' of adaptation, and furthermore, within the incalculable, intertextual elements of adaptation, how can a definitive 'whole' be quantified, or achieved? Further textual issues are raised by Boozer's argument that to fully understand 'the process of adaptation ... requires comparing the completed film with the last script draft prior to shooting' (Boozer 2008: 3-4). Whilst it can be assumed that the final shooting script will be 'closest' in content to the film produced, the studying of such a document does not necessarily illuminate the 'process' of adaptation. Indeed, in contrast to Boozer's position, it could be argued that earlier drafts, and initial responses to the source material, can tell us more about the adaptation process than a screenplay that has been honed to the point where it is fit to be used as the final, 'definitive' shooting script. Problematically, for Boozer the screenplay functions as 'the essential conceptual and creative bible for the film's construction' (Boozer 2008: 4), despite the fact that a truly definitive screenplay is unobtainable. Myriad changes can be made to a shooting script whilst in production and, most importantly, the influence of a variety of film professionals such as the editor can profoundly influence the 'tonal register' and narratological content of a film, independently of the screenplay. Boozer's desire to foreground the screenplay over the source material is problematic in as far as it elevates the screenplay to the most important text in the adaptation process, creating a new hierarchical paradigm with which to confine both adaptation and screenplay

studies. Foregrounding the screenplay, and its many drafts and forms, in this process of adaptation is also problematic due the ephemeral nature of the adapted screenplay function - that of a bridging tool, or a temporary work that catalyses source material into film.

\section{CONCLUSION}

As this essay has argued, there is significant potential for mutually beneficial, theoretical crosspollination between the fields of adaptation studies and screenplay studies. Both are preoccupied with the translatability of texts, how works exist in movement between forms, and the influences and creative decisions of practitioners. Both fields of study are formed by scholars and researchers from a wide array of inter-disciplinary backgrounds, and a broad range of critical approaches. Despite the 
relative dismissal of the screenplay's role in adaptation, two published works that illuminate the screenwriters approach to adaptation from a practice-led perspective include Marilyn Hoder-Salmon's much older, and rather prescient book, Kate Chopin's 'The Awakening': Screenplay as Interpretation (1992), and Mary H. Snyder's Analyzing Literature-to-Film Adaptations: A Novelist's Exploration and Guide (New York: Continuum, 2011). Both of these works demonstrate what can be learned from studying adaptation as a creative process through screenwriting (and vice-versa) whilst also providing discrete examples of practice-based research that enlightens our understanding of the creative remediation of source material through screenwriting. Contrastingly, in the case of Hoder-Salmon, her screenwriting project is the adapting of another writer's work, whilst Snyder evaluates the process of adapting her own novel for the screen, and offers a practice-based framework for pedagogical contexts of adaptation. These texts go some way to illustrating the mutually beneficial outcomes of adapted screenplay study, whether through textual analysis, industrial processes, or practice-based research.

In recent years scholars of screenplay studies, in general terms, have embraced both the philosophical/theoretical notions of its subject, and the sociological aspects of working screenwriters, and the industry of screenwriting. What is especially apparent from these texts is an intention to study not just the practice of screenwriting, or critical debates regarding the screenplay as text, but also significant archival work to help reveal and encourage the study of the production documents produced in the creative arts. As well as Jill Nelmes' The Screenwriter in British Cinema (2014), and Kathryn Millard's Screenwriting in A Digital Era (2014), it is perhaps Ian Macdonald's Screenwriting Poetics and the Screen Idea (2013) that has sought most earnestly to consider and take stock of the field of contemporary screenplay studies as a whole. Macdonald notes the "common interests of adaptation studies and screenwriting studies" particularly in terms of pedagogy, and in opposition to the usual tendency to privilege the role of the director over "less visible contributions" (Macdonald 2013: 224). Macdonald's analysis foregrounds the concepts of both 'screenwriting poetics': the writing and aesthetic literary qualities of the screenplays, and the 'screen idea': that very thing that screenwriters strive to write towards, outside of the final text. In this broad attempt to consider both the industrial and theoretical context of the screenplay, Macdonald galvanizes much foundational and modern screenwriting theory in order to formulate a "study of the origins, development and expression of screen ideas; and of the discourses and institutions that surround them" (Macdonald 2013: 217). 
The need to progress from comparative case-study based investigations of two 'stable' texts towards more subsidiary elements and developmental documents ephemera in adaptations is important because it reflects the demand from scholars and 'fans' for pre-versions, pre-edited first drafts, and development notes of works. This awareness and fascination for the paratextual elements of cinema can obviously lend itself to both the study and consumption of the screenplay, and access to the multifarious drafts and edited versions that exist in archives and on the internet. However, despite this increased appetite for the 'origin' document of film in screenplay studies, the in-depth study of the screenplay form is still a marginal activity in adaptation studies. The final draft of an adapted screenplay has the potential to provide us with insights into the adaptation process on one level, but delving further into previous drafts can illuminate our understanding of screenwriter's immediate, intuitive vision for the source material. For screenwriting scholars, these initial adapted screenplay drafts can also contain the traces of their literary predecessor, with detail and information that is slowly altered through subsequent drafts. This process can be seen as the active development of an adapted screenplay, as it liberates itself from the textual features of its literary antecedent, and conforms to the characteristics, and media-specific demands, of cinema. However, one of the most troublesome aspects of attempts to re-orientate adaptation studies towards the importance of the screenplay and screenwriting process is a tendency to elevate the importance of the transmedial document, and to redress the balance of its marginal status. For some the adapted screenplay is an objective document that allows the reader to understand decisions made in the adaptation process. Boozer argues that 'it is the screenplay, not the source text, that is the most direct foundation and fulcrum for any adapted film' (Boozer 2008: 4). Once again this is an interesting shift in the privileging of the dominant hypotext of adapted film. For Boozer, 'the screenplay can reveal the transformational decisions that account for a change in medium,' and can even illuminate 'the initial story and dialogue alterations that point to the conceptual goal of the film adaptation' (Boozer 2008: 9). The main problem with this argument is that it assumes a unified 'goal' for film adaptations, and that these creative decisions are revealed fullyformed in the screenplay. This essay does not argue that adaptation scholars should switch their focus solely to a textual analysis of the adapted screenplay, neither does it suggest that screenplay theorists should always analyse the industrial and creative processes of screenwriting purely as remediation. Rather, it posits cinema and television screenwriting questions of authorship that go beyond the cultural standing of the screenwriter, towards issues related to source authors of literature, comics, video games, digital texts, webisodes, and a multitude of narratives produced in our increasingly prolific convergence culture. 
The discussion of the adaptation object in this essay is also useful for considering adaptation process, and the texts that are produced within the transitional phase of pre and post production that move beyond Boozer's simplified triumvirate of source-screenplay-film. These developmental paratexts include the one-page outline, treatment, beat sheet, television series bible, shooting script, set and costume design outlines, and perhaps most pertinently, the storyboard. ${ }^{\text {iv }}$ For scholars of screenwriting and adaptation, the production of the storyboard can be seen as another paratextual stage in the adaptation process, acting as both adapted text (from the screenplay) and adaptable text (for cinematographers, designers, etc). Most interestingly, as both a visual and text based medium, the storyboard paratext acts as its own bridging stage between the literary screenplay, and audio-visual cinema. In response to Leitch's question regarding the novel as the primary source of adaptation, it is also necessary to consider the increasingly prolific use of the comic book or graphic novel as source material for adapted film and television. In these cases there is an unusually complex textual transition of a text/image based source medium, into a purely literary medium (adapted screenplay), then back into a text/image based storyboard, and then finally transitioned and edited into an audio-visual film. To analyse adaptations without recognising the importance of the various precursor texts other than the official source, is clearly doing disservice to the myriad voices and textual influences on adapted film.

The decisions made by adaptors of source material at script stage are multifarious and informed by as many practical issues as conceptual. In opposition to Boozer's view that the adapted screenplay is the most complete document of the adaptation process, it is actually the diverse, unstable and incomplete nature of the screenplay that can offer us some of the most profound insights into remediation. Rather than elevate the screenplay form to that of other literary mediums, instead it is necessary to interrogate the discrete properties of the adapted screenplay, in order to understand the characteristics that make it both peculiar and fascinating. Some of the arguments presented in this article regarding multiple authorship of literature, or literature conceived and produced consciously for the screen, or points concerning the potential literariness of the adapted screenplay, are not intended to favourably compare the form of the adapted screenplay with other media. Rather, these arguments are intended to show how all modes of storytelling are comprised of a multitude of characteristics that both complicate their stability and mediate an assembly of previous texts. The multiplicity of the adapted screenplay represents the complex relationships of texts that surround and exert various forces on adaptations. It is also the discrete nature of the screenplay form with its own poetics and narratological devices that can enable scholars of adaptation to look beyond comparative case-study analyses. The understanding of the adapted screenplay as a mediated, and interstitial form in the process of transition 
can enable adaptation studies to identify adapting as a process, and one that can be separated from the source material, or final film. For screenwriting studies, the concept of creative process as the adaptation of previous source texts, can enable fruitful studies of canonicity, intertextualty, authorship and the role of the screenwriter as remediator of limitless precursor texts. It is perhaps in the creative and industrial spaces between works in transition, and the performances of remediation and retelling outside of source text and adapted text, which can provide the most fruitful areas of cross-over for these two developing and vibrant fields of study.

\section{References}

Boon, K.A. (2008), Script Culture and the American Screenplay, Detroit, MI: Wayne State University Press.

Boozer, J. (2008), 'Introduction: The Screenplay and Authorship in Adaptation', in J. Boozer (ed.), Authorship in Film Adaptation, Austin: University of Texas Press, pp.1-30.

Cartmell, D. and I. Whelehan (eds.). (1999), Adaptations: from Text to Screen, Screen to Text. London: Routledge.

(2010), Screen Adaptation: Impure Cinema, Houndmills: Palgrave.

Elliott, K. (2003), Rethinking the Novel/Film Debate. Cambridge: Cambridge University Press.

Geraghty, C. (2008), Now a Major Motion Picture: Film Adaptations of Literature and Drama, Lanham, MD: Rowman and Littlefield.

Hoder-Salmon, M. (1992), Kate Chopin's 'The Awakening': Screenplay as Interpretation, Gainesville: University Press of Florida.

Horvath, A., B. Thomassen and H. Wydra (2009), 'Introduction: Liminality and Cultures of Change', International Political Anthropology, 1: 2, pp. 255-261.

Hutcheon, L. (2006), A Theory of Adaptation, London: Routledge.

Kohn, N. (1999), 'Disappearing Authors: A Postmodern Perspective on the Practice of Writing for the Screen', Journal of Broadcasting \& Electronic Media, 43: 3, pp. 443-449.

Leitch, T. (2005), 'Twelve Fallacies in Contemporary Adaptation Theory', Criticism, 45: 2, pp. 149171.

(2007), Film Adaptation and Its Discontents: From Gone with the Wind to The Passion of the Christ, Baltimore: Johns Hopkins University Press.

- (2008), 'Adaptation, The Genre', Adaptation, 1: 2, pp. 106-120.

Macdonald, I.W. (2004), 'Manuals Are Not Enough: Relating Screenwriting Practice to Theories', Journal of British Cinema and Television, 1:2, pp. 260-274.

- (2013), Screenwriting Poetics and the Screen Idea, Basingstoke: Palgrave Macmillan.

Maras, S. (2009), Screenwriting: History, Theory and Practice, London and New York: Wallflower Press. 
McMurtry, L. (1987), Film Flam: Essays on Hollywood, New York: Simon and Schuster.

Murray, S. "'Phantom Adaptations: Eucalyptus, The Adaptation Industry and the Film That Never Was,." Adaptation 1.1 (2008): 5-23.

(2011), The Adaptation Industry: The Cultural Economy of Contemporary Literary Adaptation, London: Routledge.

Nelmes, (2011), Analysing the Screenplay, London \& New York: Routledge.

Price, S. (2010), Screenplay: Authorship, Theory and Criticism, Hampshire: Palgrave Macmillan.

Rilla, W. (1973), The Writer and the Screen: On Writing for Film and Television, London and New York: W.H. Allen.

Sherry, J. (2014), 'Teaching Adapting Screenwriters: Adaptation Theory through Creative Practice', in D. Cartmell and I. Whelehan (eds.), Teaching Adaptations, Basingstoke: Palgrave Macmillan, pp. 87-105.

Snyder, M. H. (2011), Analyzing Literature-to-Film Adaptations: A Novelist's Exploration and Guide, New York: Continuum.

Stam, R. (2004), 'Introduction: The Theory and Practice of Adaptation' in R. Stam and A. Raengo (eds.), Literature and Film: A Guide to the Theory and Practice of Film Adaptation, Oxford: Blackwell, pp. 1-52.

Sternberg, C. (1997), Written for the Screen: The American Motion-Picture Screenplay as Text, Tübingen: Stauffenburg-Verl.

Turner, V. (1967), 'Betwixt and Between: The Liminal Period in Rites de Passage', in V. Turner, The Forest of Symbols, Ithaca, NY: Cornell University Press, pp. 46-55. (1969), The Ritual Process: Structure and Anti-Structure, New York and London: Aldine.

Verevis, C. (2005), Film Remakes, Edinburgh: Edinburgh University Press.

Woolf, V. (1926), 'The Cinema', in Arts, June 1926, pp. 314-316.

i

The subject of remakes is studied with a great deal of rigour in Constantine Verevis' Film Remakes (Edinburgh: Edinburgh University Press, 2005).

ii

Not only do Rilla's gendered pronouns date his work, they also raise issues about authorship and the profession of screenwriting. For more on the gender disparity in screenwriting in the UK, see the UK Film Council report "Scoping Study into the Lack of Women Screenwriters in the UK", commissioned from Institute for Employment Studies, 2006. http://www.ukfilmcouncil.org.uk/media/pdf/4/r/0415womenscreen_-_FINAL_09.06.06.pdf. 2006.

iii

In particular, writers such as Mickey Spillane, Raymond Carver and Cormac McCarthy who purposefully avoid florid language in favour of a more 'sparse' narrative style.

iv

This author looks forward eagerly to the currently unpublished Storyboarding: A Critical History (Palgrave Macmillan, 2015) by Chris Pallant and Steven Price, as an example of this inquiry into the creative and industrial texts produced during film production. 